\title{
The Short-Term Effect of Online Violent Stimuli on Aggression
}

\author{
Jingjin Tian"1\#, Qian Zhang1,2,3,4\#, Jian Cao4, Philip Rodkin' \\ ${ }^{1}$ School of Applied Technology, Southwest University, Chongqing, China \\ ${ }^{2}$ Department of Psychology, Southwest University, Chongqing, China \\ ${ }^{3}$ Center for the Study of Mental Health Education, Southwest University, Chongqing, China \\ ${ }^{4}$ Research Institute of Calligraphy, College of Literature, Southwest University, Chongqing, China \\ ${ }^{5}$ Department of Educational Psychology, University of Illinois, Champaign, IL, USA \\ Email: ${ }^{2}$ zhq@swu.edu.cn
}

Received 22 October 2015; accepted 4 March 2016; published 7 March 2016

Copyright (C) 2016 by authors and Scientific Research Publishing Inc.

This work is licensed under the Creative Commons Attribution International License (CC BY).

http://creativecommons.org/licenses/by/4.0/

c) (i)

\section{Abstract}

The significance of this study was to find whether violent stimuli exposure could escalate the following levels of aggression in order to better cultivate aggression education among contemporary undergraduates in China. This study mainly tested the effects of violent stimuli on aggression by employing modified Stroop task. A total of 188 undergraduates participated in this study. Re- sults showed that undergraduates exposing to violent stimuli exhibited high levels of aggression, whereas undergraduates who exposed to non-violent stimuli displayed low levels of aggression. Specifically, males, but not females, manifested high levels of aggression after exposure to violent sti- muli. Moreover, viewers with high-aggressiveness (HA), but not with moderate-aggressiveness (MA) and low-aggressiveness (LA), showed high levels of aggression after exposure to violent stimuli via internet.

\section{Keywords}

Violent Stimuli, Aggression, Undergraduates, Modified Stroop Task

\section{Introduction}

Online violent stimuli were referred to any medium forms of violent materials online, including movies, pictures, cartoons, video games, music, books, newspapers, magazines, CDs, DVDs, and videotapes. Over the past few years, numerous evidences accordingly implied that exposure to online violence promoted aggression. For in-

\footnotetext{
*Corresponding author.

"Both Qian Zhang and Jingjin Tian were the first authors because of their equal contribution to this article
} 
stance, a series of studies carried out by prior researchers observed that many kids became aggressive towards a Bobo doll after exposing violence [1]. The research demonstrated that young children imitated the model, especially if it was the same sex as them. Further, Bandura demonstrated that children saw movies of people being aggressive in which the aggression was rewarded, punished, or not shown to have any consequences, they might do more aggressive things in their daily life. Besides, children's sensitivity might be reduced to violent acts (i.e., anti-social behavior) due to long-term exposure to online violent stimuli. In other words, this reduced sensitivity occurs if people repeatedly view violent acts and can be empirically shown by reduced physiological arousal in those who have previously viewed violence as compared to those who have not seen it. Researchers assumed that children who had previously seen a violent videotaped program became less physiologically aroused by an aggressive videotape than those who had not seen the program [2].

Internet media violence, to some extent, was regarded as special violent stimuli which affected adolescents' lives via television and the movies [3]. There were many theoretical references that media violence might have larger effect on aggression than media non-violence. As such, some eyebrows were raised questions like whether youth should watch violent movies or play such violent games. In general, several theories could interpret the aggression formation and reasons underlying the effects of media violence on aggression. General Aggression Model (GAM) specified the short-term effects of violent video games on aggression, and that exposure to violent video games increased aggressive behavior in the short run (within 20 minutes of game play). GAM theoretical approach emerged from prior researchers' work on various aggression-related domains, and integrated existing theory and data regarding the acquirement, instigation, and expression of human aggression. Specifically, it was noted that aggression was largely based on cognitive structures (scripts, schemas) created by social learning processes. Thus, it incorporated the theoretical insights of much previous theories of aggression, especially Social Learning Theory, Cognitive Neoassociation Model [4], Social Information-processing Model [5], and Social-Cognition Model. GAM described a multistage process by which personal (e.g., aggressive personality) and situational (e.g., video game play and provocation) input variables led to aggressive behavior. Importantly, they did so by influencing several related internal states and the automatic and controlled appraisal or decision process. In our points of view, input variables, such as violent stimuli, impacted individuals' current affection, such as aggressive feelings or hostility. Cognition, affect, and physical arousal were seen as highly interrelated aspects of one's current internal state. In sum, short-term violent video game increased levels of aggression were expected by GAM whenever exposure to violent media primed aggressive thoughts, hostile feelings, and increases arousal. Long-term media violence effects on aggression resulted from the development and reinforcement of aggression-related knowledge structures. Each time people exposing to violent media, they rehearsed aggressive scripts that reinforced vigilance for enemies, aggressive action against others, expectations that others would behave aggressively. What's more, repeated exposure to violent scenes was likely to be desensitizing in these aggression-related knowledge structures, and then the desensitization effects changed the individual's personality.

Although much research examined the effects of movie and game violence, the empirical literature on online media violence was sparse. To what extent did the media violence support or contradict the GAM-based hypothesis? For the measurement of aggression induced by media violence, previous studies used self, teacher, and peer reports to do this. However, none of them distinguished between violent and nonviolent games. Thus, none tested the hypothesis that media violence was uniquely associated with escalated aggression.

Noticeably, none of these studies could rule out the possibility that key variables such as excitement, difficulty, or enjoyment created the increase in aggression. From previous researches [6], violent stimuli (materials) tended to be more exciting than nonviolent materials, so the effects could be the result of higher levels of aggression induced by violent stimuli rather than by non-violent stimuli. In addition, researchers found that 3rdand 4th-graders assigned to play a violent game gave more hostile interpretations for a subsequent ambiguous provocation story than children assigned to play a nonviolent game [7]. Nevertheless, other studies of media violence showed no effect on aggression [8]. Interestingly, little evidence showed that the violent content of media could increase aggression immediately.

\section{The Present Research}

The study was to lay firm empirical foundation for understanding violent stimuli effects online, in order to provide sounding evidence for GAM theories. We used undergraduate population, in part because they are old 
enough for short-term effects of watching violent movies to have had a measurable impact on real-world aggression. Additionally, we randomly assigned participants to watch either a violent or a nonviolent movie as stimuli; the two movies were matched on several key dimensions. Subsequently, these participants completed Stroop task in which they could react to the stimuli rapidly and accurately. We also assessed the subjective feelings of aggression induced by media violence. Meanwhile, we examined the individual variables of trait aggressiveness, one indicator of aggressive personality, as well as gender differences. These variables have yielded interesting effects in several media violence studies [8] [9]. In this study, we attempted to examine the short influence of online violent stimuli on aggression among undergraduates and underlying mechanisms.

\section{Method}

\subsection{Participants}

188 Chinese undergraduates (50\% females) were randomly selected to participate in the study. They ranged in age from 19 to 23 years $(M=21.32, S D=1.23)$, with 94 designated to violent group (experimental group) and 94 distributed to non-violent group (control group). They were right-handed, no color blindness, without any physical and mental disorders.

\subsection{Stimuli Selection}

We selected violent-and non-violent movies as Stimuli (Materials). Ninja Assassin ${ }^{1}$, an American violent film, directed by James Mc Teigue, was produced by Joel Silver and the Wachowski brothers. Ninja Assassin explored political corruption, child endangerment, and the impact of violence. It was commercially distributed by Warner Brothers Pictures. We chose 15-min Ninja Assassin clip, which consists of body fighting scenes and verbal attacks used in the experiment. Hence, it could be deemed as violent stimuli, and it was in agreement with the definition of violence [2]. Big Nothing ${ }^{2}$, an European blockbusters, was directed by Jean-Baptiste Andrea. The film was set in a small Oregon town, where a brutal serial killer nicknamed "Oregon undertaker" had been murdering and mutilating young women. This movie included no body fighting scenes and verbal attacks. Therefore, this 15-min Big Nothing clip could be regarded as non-violent stimuli.

\subsection{Goal Words}

50 aggressive (e.g., assault, stab) and 50 nonaggressive words (e.g., smile, walk) were randomly matched. The words were presented in italics (font size $=72$ ) in one of three colors (green, red, or yellow) in the screen center with gray background.

\subsection{Measures}

Buss-Perry Aggression Questionnaire (BPAQ), a 5-point Likert scale, was used to measure aggressive personality. BPAQ included four dimensions: physical aggression (PA), verbal aggression (VA), anger (A), and hostility $(\mathrm{H})$. Some items were reverse coded to ensure higher scores, indicating higher aggressive personality. Cronbach's alpha coefficient is a measure of internal consistency, for which the scale was 0.94 . A measure of testretest reliability yielded a correlation coefficient of 0.89 . The values of the Cronbach's alpha coefficients for physical aggression, verbal aggression, anger, and hostility were $0.85,0.72,0.83$, and 0.77 , respectively. The reliability of this scale for this study was acceptable [10]. Participants who obtained the top 1/3 of the BPAQ scores were regarded as HAP, and those obtaining the bottom 1/3 were deemed as LAP.

\footnotetext{
${ }^{1}$ Ninja Assassin focused on the character Raizo (Rain), who was raised by the Ozunu Clan to become the most lethal ninja assassin in the world. As a child, Raizo (since he was an orphan) was taken in by Lord Ozunu (Sho Kosugi) and was subjected to a severely brutal training to become the next successor of their clan. As a result of Kiriko's death, Raizo began to resent and doubt Lord Ozunu. The scene selected involved a disguised female assassin (Linh Dan Pham) who attempted to kill Raizo at a laundromat; the scene involves attacks and fierce killing.

${ }^{2}$ Big Nothing told the story of Dr. Charlie (played by David Schwimmer) who was in trouble. Charlie lost his job at the university. To ensure he was not a burden to his family, Charlie found a telephone sales job. It is there that he met his former colleague, Garth (played by Simon Pegg). Garth told Charlie his plan, namely to blackmail a regular browser of a pornographic website. To allow the family to live a rich life, Charlie agreed to join. The two were joined by a teenage pageant queen Josie McBroom (played by Alice Eve). The three began this scheme The experimental scene we selected was a fragment of the humorous movie.
} 


\subsection{Research Design}

A multi-factorial design was employed to examine the main effect and interaction among variables. A 2 (Stimuli type: violent, non-violent) $\times 2$ (Goal word: aggressive, nonaggressive) $\times 2$ (Grade: freshmen, sophomore) $\times 2$ (Aggressive personality: HAP, LAP) four repeated measures analysis of variance (ANOVA) was conducted, with stimuli type, grade and aggressive personality as the between-group factor, and goal word as the within-group factor. Stimuli type was the independent variable, and aggression was the dependent variable.

\subsection{Procedure}

All the participants were consented to attend the experiment. First, they completed the BPAQ. Second, they were randomly assigned to watch either the violent-or non-violent movie clip. Third, they completed a modified Stroop task programmed by E-prime software. Participants were told that it was a test of their speed and accuracy of responses, and the goal words would be presented in different colors (red, yellow, or blue). If the word color was red, they pressed "1" on keyboard; If the word color was yellow, they pressed "2"; If the word color was blue, they pressed " 3 ". Participants should react as quickly and accurately as possible, and distinguish the word color regardless of word meaning. After the instructions appeared, a plus sign (i.e., +) was presented at the center of the screen for $300 \mathrm{~ms}$. Then, the goal words were presented at the center of the screen for 1, 000ms. The inter-stimulus interval (ISI) was 200 to 300 ms. After participants reported the word color, the program proceeded to the next trial. If participants did not respond in $1000 \mathrm{~ms}$, the program proceeded to the next trial automatically (see Figure 1). Each participant's accuracy rate and reaction time (RT) were recorded. When the session was over, concluding remarks appeared on the screen and participants were debriefed.

The Stroop procedure was divided into two sections: practice session and formal session. The practice session consisted of 20 trials which did not appear in the subsequent formal session. The program returned to the practice session if the accuracy rate was less than 85 percent. The practice session aimed to familiarize the participants with key pressing, and to counterbalance the color and key pressing. A formal session was divided into four blocks. There were 25 trials per block. Thus, the total number of trials was 100.50 aggressive and 50 nonaggressive words were presented in one of three colors (red, yellow, or blue), and each word appeared once per block. After a short rest between the blocks, the program proceeded to the next block. The mean accuracy rate of the participants was from 85 percent to 95 percent.

\section{Results}

\subsection{Main Effect of Goal Word on Levels of Aggression}

One-way analyses of variance (ANOVAs) were performed to examine the main effect of goal word on aggression (see Table 1). As expected, significant shorter reaction time (RT) to aggressive words (504.27 ms) than to nonaggressive words $(576.44 \mathrm{~ms})$ was found after violent movie exposure $[F(1,66)=4.18, P<0.05]$.

\subsection{AAS Difference between Violent and Non-Violent Groups}

Based on the significant longer RT to nonaggressive words than to aggressive words (see Table 1), we created the aggressively activated score (AAS) to better illuminate the priming effects of violent movie on aggression. AAS could be calculated by subtracting the RT to nonaggressive words from the RT to aggressive words. As shown in Table 2, the RT to aggressive words was shorter than to nonaggressive words ( $501.25 \mathrm{~ms}<566.35 \mathrm{~ms}$ ) when violent movie was presented. However, the RT to aggressive words was a bit longer than to nonaggressive words (525.24 ms > $518.89 \mathrm{~ms}$ ) while non-weapon pictures appeared. Thus, the AAS for violent and non-violent groups were $-65.10 \mathrm{~ms}$ and $6.35 \mathrm{~ms}$, respectively.

\subsection{Multivariate Analysis of Covariance (MANCOVA) for Stimuli Type and Goal Word}

To further examine whether the AAS yielded statistically significant differences for independent and dependent variables, MANCOVA was carried out (see Table 3). As can be seen, no main significant differences were found for stimuli type $[F(1,54)=0.48, P>0.05]$, suggesting that the RT to goal words was not significant under each condition of prime presentation. A significant stimuli type $\times$ gender interaction was found $[F(1,157)=$ $1.67, P<0.01]$, and further simple effect analysis indicated that AAS of males was significantly smaller when 


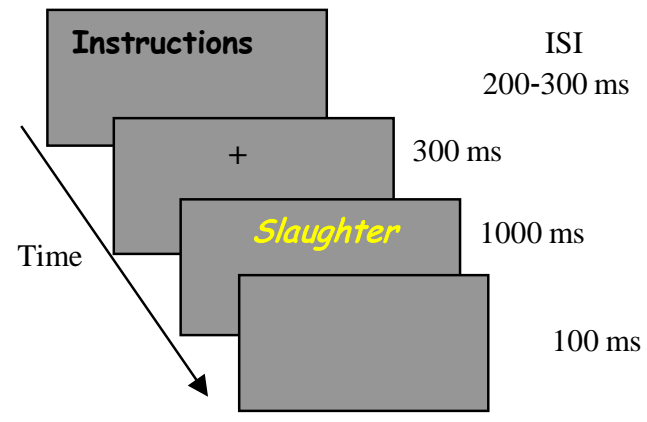

Figure 1. Modified Stroop task (Note: Modified Stroop task was to test participants' RT to the color aggressive words and nonaggressive words by pressing keys on keyboard during a specified time).

Table 1. RT differences for goal words after violent stimuli exposure.

\begin{tabular}{ccrrrr}
\hline \multicolumn{5}{c}{ Goal words } \\
& \multicolumn{2}{c}{ Aggressive words } & \multicolumn{2}{c}{ Non-aggressive words } & $F$ \\
& $M$ & $S D$ & $M$ & $S D$ & \\
RT & 504.27 & 549.27 & 576.44 & 52.61 & $4.18^{*}$ \\
\hline
\end{tabular}

Note: $\mathrm{RT}=$ reaction time; $* P<0.05$.

Table 2. Mean RT (milliseconds) to aggressive and nonaggressive words.

\begin{tabular}{cccccc}
\hline & \multicolumn{5}{c}{ Goal words } \\
\hline Stimuli & \multicolumn{2}{c}{ Aggressive words } & \multicolumn{2}{c}{ Nonaggressive words } & AAS \\
& $M$ & $S D$ & $M$ & $S D$ & -65.10 \\
Violent movie & 501.25 & 81.03 & 566.35 & 84.29 & 79.98 \\
Non-violent movie & 525.24 & 80.21 & 518.89 & 6.35 \\
\hline
\end{tabular}

Note : RT = reaction time; AAS = aggressively activated score; AAS = mean RT to violent movie minus RT to non-violent movie.

Table 3. MANCOVA for AAS among research variables.

\begin{tabular}{cccc} 
Variables & $M$ & $S D$ & $F$ \\
Stimuli type & 338.27 & 53.45 & 0.48 \\
Stimuli type $\times$ Gender & 1448.96 & 154.33 & $1.67^{* *}$ \\
Stimuli type $\times$ aggressive personality & 1632.08 & 187.62 & $1.78^{* *}$ \\
\hline
\end{tabular}

Note: $* * P<0.01$.

exposing to violent stimuli than when exposing to non-violent stimuli $[(F(1,210)=3.41, P<0.01]$, but no significant difference for AAS of females $[F(1,118)=1.07, P>0.05$; see Table 4). Furthermore, a significant movie type $\times$ aggressive personality interaction was found $[F(1,227)=1.78, P<0.01$; see Table 3], and further simple effect analysis demonstrated that AAS of participants with HAP was significantly smaller when exposing to violent stimuli than when exposing to non-violent stimuli $[F(1,245)=8.14, P<0.05]$, whereas AAS of participants with LAP was not significant $[F(1,94)=0.36, P>0.05$; see Table 5] under conditions of viewing violent-and non-violent movies. No significant interaction, however, was found among movie type, aggressive personality, and gender $[(F=6.43, P>0.05$; see Table 3$]$.

\section{Discussion}

\subsection{Violent Stimuli Exposure Could Significantly Elevate Aggression}

Overall, significant shorter RT to aggressive words than to nonaggressive words was found, implying that violent stimuli significantly activated aggression among undergraduates. This result supported our first hypothesis, 
Table 4. Post hoc test of AAS difference for males and females.

\begin{tabular}{cccc}
\hline Gender & Violent movie & Non-violent movie & $F$ \\
\hline Males & -5.27 & 9.24 & $3.41^{* *}$ \\
Females & 4.58 & 10.98 & 1.07 \\
\hline
\end{tabular}

Note: $* * P<0.01$.

Table 5. Post hoc test of AAS difference for aggressive personality.

\begin{tabular}{cccc}
\hline Aggressive personality & & Non-violent movie & $F$ \\
\hline HAP & -2.75 & 17.95 & $8.14^{*}$ \\
LAP & -342 & -0.74 & 0.36
\end{tabular}

Note: HAP = highly aggressive personality, LAP = lowly aggressive personality. ${ }^{*} P<0.05$.

and it was correspondent with previous study [11]. Some experts assumed contextual factors were responsible for aggressive behavior after viewing violent movies [2]. Accordingly, some researchers contended that repeatedly gun violence or media violence exposure could decrease empathy and desensitization to aggression [12] [13]. So, we can speculate that individuals were prone to be aggressive because of aggressively cognitive schema activated in neural mechanisms. In fact, college students consumed lots of attentional and cognitive resources to identify violent stimuli and subsequent aggressive words, activating their aggressive schema. Thus, violent stimuli, such as movie violence, might cause hostile and violently semantic information derived from short-term and long-term memory. Based on this, we could assume that variant aggressive knowledge of cognitive structure or cognitive schema led to undergraduates' short-term effect of aggression at least. Importantly, this kind of viewpoint could be verified from General Aggression Model (GAM) and Cognitive Neassociation Model, which all underlie the facts that repeated exposure to violent stimuli strengthened aggressive cognition other than non-violent stimuli.

\subsection{Violent Stimuli Exposure Apparently Elicited Levels of Aggression among Males}

As we can see, only males’ aggression was significantly strengthened after violent stimuli exposure, which, confirmed the hypothesis 2, and it was also supported the results of prior researches [14] [15]. For this reasons of phenomenon, some researchers argued that males have more sensitively cognitive framework of aggression than females when exposing to violent stimuli [16] [17]. So, sex differences in processing words relating to direct and indirect aggression again demonstrated that males showed a perceptual bias for words regarding direct aggression, and males spent significant longer time to name color of aggressive words than females [18]. Upon these researches, we could infer that gender difference could be a good predictor to show different levels of aggression after exposing to violent stimuli.

Currently, males were more eager to watch violent movie than females. Some scholars found that males tended to display physical aggression, whereas females exhibited relational aggression [19]. Specifically, females like to feel more distressed than males when they were depressed and anxious. In particular, researchers told that males regarded bulling as the symbol of high social and economic status, showing bulling behavior upon others [20]. In addition to that, males regarded their roles as dominant, whereas females could have the capability of self-control over aggression. In the daily life, females were likely to hide their true behavior and share innate feelings with friends when they fell into feeling of aggression. On the contrary, males preferred to alleviate the negative emotion by conducting violent behavior. Thus, we could infer that gender differences of aggression after exposing to violent stimuli were in part linked to neuro-biological, cultural, and personality factors, indicating that neural variable, cognition and aggressive cognition, hostile emotion and aggressive behavior.

\subsection{Violent Stimuli Exposure Induced Levels of Aggression among Undergraduates with HAP}

In this study, aggression of undergraduates with HAP was significantly induced after violent stimuli exposure. Aggression of those with LAP, nevertheless, was not significantly activated. This result was in agreement with 
hypothesis 3. And it also replicated previous studies [21] [22]. For participants with HAP, they might be a type of special group who had lots of problems of psychological adaptability [23]. In the process of adaptability on campus, the HAP might cause them to show more aggressive cognition and aggressive behavior than the people with LAP and HAP. Hence, there was a probability that the cognitive networks of undergraduates with HAP were more easily strengthened by violent stimuli rather than those with LAP, implying that different personality traits was linked to levels of aggression. For the deep reasons, undergraduates with HAP might have faster aggressively neural association networks in brain than those with LAP, indicating that undergraduates with HA were more susceptible to violent stimuli relative to those with LAP. The aggressive personality might be a vitally important individual factor to impact levels of aggression among Chinese undergraduates after exposing to violent stimuli instead of non-violent stimuli.

\subsection{Limitations}

Some limitations should be noticed in our study. First of all, the selected sample was relatively consubstantial. As such, generalized validity should be doubted when applying conclusions to other groups (i.e., cross-cultures, races). Secondly, using cross-sectional design might limit the clarification of the casual correlations among independent and dependent variables explored in this study. Thirdly, careful selection of violent stimuli should be regarded as a further step. In our study, we just select violent movies as violent stimuli, the more accurate selective standards and expert consultation should be carefully considered in future research of aggression among Chinese undergraduates.

\section{Acknowledgements}

This study was funded by "the Fundamental Research Funds for the Central Universities" (Grant Number: SWU1409135), and it was also supported by the Youth Research Grant of Social Science and Humanities Project from the Ministry of Education in China (Grant Number: 13YJC190030), as well as "the Fundamental Research Funds for the Central Universities” (Grant Number: XDJK 2016C077) and Ph. D funds from “the Fundamental Research Funds for the Central Universities” (Grant Number: SWU 1509528). We are indebted to each undergraduate who participated in the study as well as the anonymous reviewers for their kind feedback on previous drafts of this manuscript.

\section{References}

[1] Bandura, A. (1965) Influence of Models' Reinforcement Contingencies on the Acquisition of Imitative Responses. Journal of Personality and Social Psychology, 1, 589-595. http://dx.doi.org/10.1037/h0022070

[2] Anderson, C.A. and Bushman, B.J. (2001) Effects of Violent Video Games on Aggressive Behavior, Aggressive Cognition, Aggressive Affect, Physiological Arousal, and Prosocial Behavior: A Meta-Analytic Review of the Scientific Literature. Psychological Science, 12, 353-359. http://dx.doi.org/10.1111/1467-9280.00366

[3] Huesmann, L.R. (2010) Nailing the Coffin Shut on Doubts That Violent Video Games Stimulate Aggression: Comment on Anderson et al. (2010). Psychological Bulletin, 136, 179-181. http://dx.doi.org/10.1037/a0018567

[4] Berkowitz, L. (2003) Affect, Aggression, and Behavior. In: Davidson, R.J., Scherer, K.R. and Goldsmith, H.H., Eds., Handbook of Affective Science, Oxford University Press, New York, 804-823.

[5] Coie, J.D., Dodge, K.A., Terry, R. and Wright, V. (1991) The Role of Aggression in Peer Relations: An Analysis of Aggression Episodes in Boys’ Play Groups. Child Development, 62, 812-826. http://dx.doi.org/10.2307/1131179

[6] Tian, J.J. and Zhang, Q. (2014) Are Boys More Aggressive than Girls after Playing Violent Computer Games Online? An Insight into an Emotional Stroop Task. Psychology, 5, 27-31. http://dx.doi.org/10.4236/psych.2014.51006

[7] Kirsh, S.J. (2003) The Effects of Violent Video Game Play on Adolescents: The Overlooked Influence of Development. Aggression and Violent Behavior, 8, 377-389. http://dx.doi.org/10.1016/S1359-1789(02)00056-3

[8] Elson, M. and Ferguson, C.J. (2013) Does Doing Media Violence Research Make One Aggressive? The Ideological Rigidity of Social Cognitive Theories of Media Violence and Response to Bushman and Huesmann (2013), Krahé (2013), and Warburton (2013). European Psychologist, 19, 68-75. http://dx.doi.org/10.1027/1016-9040/a000185

[9] Anderson, C.A. and Huesmann, L.R. (2003) Human Aggression: A Social-Cognitive View. In: Hogg, M.A. and Cooper, J., Eds., The Sage handbook of social psychology, Sage, Thousand Oaks, CA, 296-323.

[10] Bushman, B.J. and Huesmann, L.R. (2010) Aggression. In: Fiske, S.T., Gilbert, D.T. and Lindzey, G., Eds., Handbook of Social Psychology, 5th Edition, John Wiley \& Sons, New York, 833-863. 
http://dx.doi.org/10.1002/9780470561119.socpsy002023

[11] Buss, A.H. and Perry, M.P. (1992) The Aggression Questionnaire. Journal of Personality and Social Psychology, 63, 452-459. http://dx.doi.org/10.1037/0022-3514.63.3.452

[12] Bartholow, B.D., Anderson, C.A., Carnagey, N.L. and Benjamin, A.J. (2005) Interactive Effects of Life Experience and Situational Cues on Aggression: The Weapons Priming Effect in Hunters and Nonhunters. Journal of Experimental Social Psychology, 41, 48-60. http://dx.doi.org/10.1016/j.jesp.2004.05.005

[13] Holden, C. (2005) Controversial Study Suggests Seeing Gun Violence Promotes It. Science, 308, 1239-1240. http://dx.doi.org/10.1126/science.308.5726.1239a

[14] Strasburger, V.C. and Wilson, B.J. (2002) Children, Adolescents, and the Media. Sage, Thousand Oaks, CA.

[15] DeWall, C.N., Anderson, C.A. and Bushman, B.J. (2011) The General Aggression Model: Theoretical Extensions to Violence. Psychology of Violence, 1, 245-258. http://dx.doi.org/10.1037/a0023842

[16] Boutwell, B.B., Franklin, C.A., Barnes, J.C. and Beaver, K.M. (2011) Physical Punishment and Childhood Aggression: The Role of Gender and Gene-Environment Interplay. Aggressive Behavior, 37, 559-568. http://dx.doi.org/10.1002/ab.20409

[17] Smith, P. and Waterman, M. (2005) Sex Differences in Processing Aggression Words Using the Emotional Stroop Task. Aggressive Behavior, 31, 271-282. http://dx.doi.org/10.1002/ab.20071

[18] Conley, C.S. and Rudolph, K.D. (2009) The Emerging Sex Difference in Adolescent Depression: Interacting Contributions of Puberty and Peer Stress. Development and Psychopathology, 21, 593-620. http://dx.doi.org/10.1017/S0954579409000327

[19] Rose, A. and Rudolph, K.D. (2006) A Review of Sex Differences in Peer Relationship Processes: Potential Trade-Offs for the Emotional and Behavioral Development of Girls and Boys. Psychological Bulletin, 132, 98-131. http://dx.doi.org/10.1037/0033-2909.132.1.98

[20] Cross, C.P. and Campbell, A. (2012) The Effects of Intimacy and Target Sex on Direct Aggression: Further Evidence. Aggressive Behavior, 38, 272-280. http://dx.doi.org/10.1002/ab.21430

[21] Rodkin, P.C. and Berger, C. (2008) Who Bullies Whom? Social Status Asymmetries by Victim Gender. International Journal of Behavioral Development, 32, 473-485. http://dx.doi.org/10.1177/0165025408093667

[22] Wilson, T. and Rodkin, P.C. (2013) Children's Cross-Ethnic Relations in Elementary Schools: Concurrent and Prospective Associations between Segregation and Social Status. Child Development, 84, 1081-1097. http://dx.doi.org/10.1111/cdev.12020

[23] Zhang, D.J. and Zhang, Q. (2007) Developmental Characteristics of Adaptability among College Students in China. Journal of Southwest University (Social Sciences Edition), 33, 124-128. 